\title{
A Report of a Worker Suffering from Pneumatosis Cystoides Intestinalis Following Trichloroethylene Exposure
}

\author{
Key words: Trichloroethylene-Trichloroacetic acid-Intestinal cysts-Pneuma- \\ tosis cystoides intestinalis-Gas chromatography-Mass spectrometry
}

Pneumatosis cystoides intestinalis (PCI) is characterized by the presence of gaseous cysts in the colonic wall. It is an uncommon disease for which the pathogenesis is still obscure and remains under debate. PCI was first described in the eighteenth century (Jamart et al. 1979). ${ }^{1)}$ Reviews of this disease in 255 cases were published by Koss. ${ }^{2)}$

Recently, the occurrence of PCI in workers exposed to trichloroethylene (TRI) has been reported by Japanese researchers. ${ }^{3-7)}$ Histological studies of this disease showed that the cysts were lymphatic vessels distended with gas (Koss 1952). ${ }^{2)}$ PCI is classified into two categories: Secondary PCI associated with diseases such as gastrointestinal abnormalities and obstructive pulmonary diseases, and primary (idiopathic) PCI without significant complications.

Yamaguchi $^{3)}$ reported in 1985 that primary PCI is related to TRI exposure based on the results of 11 positive cases out of 12 cases of PCI which had been diagnosed in the Department of Internal Medicine, Shinshu Medical School, and in associated hospitals. Case reports of PCI patients who were exposed to TRI have been reported ${ }^{4-6)}$ since then. An epidemiological study by Sato ${ }^{7)}$ showed that 15 of 21 cases of primary PCI were exposed to TRI.

The presence of trichloroacetic acid (TCA) in urine of a patient was demonstrated by GC-MS. Concentrations of TCA in bile and urine of the PCI patient were determined by colorimetry.

A 23-year-old male lathe operator had been soaking metal parts in warm TRI in a tank under ultrasonic waves to degrease them after planing. Environmental air was collected in a Tedlar-bag at 5 sampling points in a unit work area around the degreasing tank where he had been working for more than 5 years. The general air concentration of TRI was determined by gas chromatography using PEG $20 \mathrm{M}$ on a chromosorbe column. The geometric mean and $90 \%$ range of TRI concentration were $32 \mathrm{ppm}$ and $18-56 \mathrm{ppm}$, respectively. In addition, he had been engaged in the washing process for about 1 minute at least once a day for 5 years. During this procedure he was exposed to a higher concentration of TRI than in the general air of his working environment. Exposure to TRI continued until August 25, 1987. The patient had constipation from February to April 1985, but this disappeared spontaneously. Later, in May 1987, he again suffered from severe constipation with defecation once a week. At that time he had abdominal fullness without abdominal pain. He did not excrete 


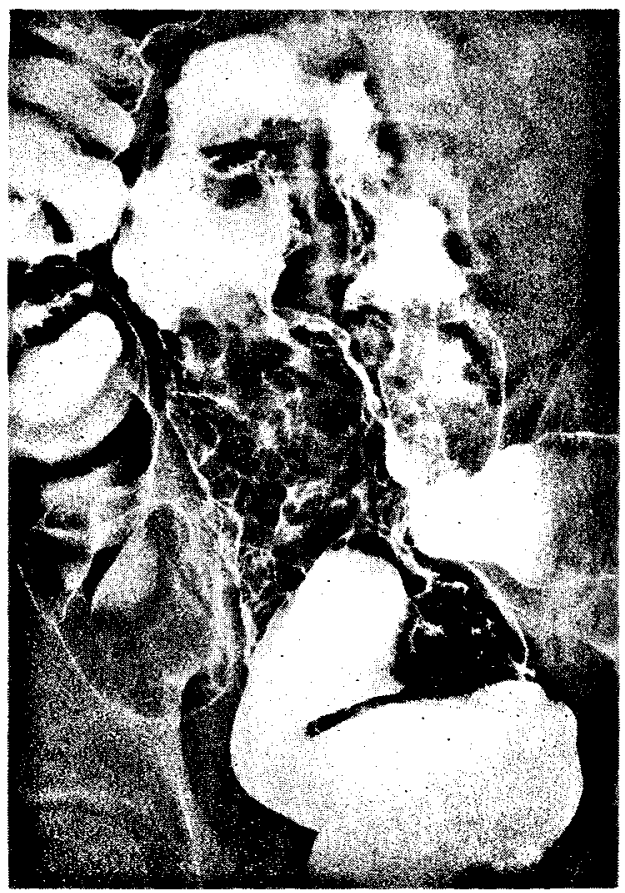

Fig. 1. Double-contrast barium enema demonstrating multiple polypoid lesions in the sigmoid colon. (photograph by Kihara and Kamoi)

blood in the stool. As the complaints did not subside, he sought admission to the Department of Gastroenterology, Kawasaki Medical School Hospital on August 26, 1987. During hospital treatment, the worker was not exposed to trichloroethylene. He had no history of severe disease except appendecitis, for which he underwent appendectomy at the age of 14 years. He was often constipated but did not have hematochezia. He had no particular family history of disease. Characteristic honeycomb gas shadows were recognized on plain $\mathrm{x}$-ray film, and the diagnosis of PCI was confirmed by barium enema (Fig. 1) and colonfiberscopic examination. Cysts were detected in the ascending and sigmoid colons by barium enema. Intestinal cysts disappeared with hyperbaric oxygen $(50 \%)$ inhalation therapy given from September 11 to 20 . Disappearance was confirmed by barium enema. His constipation was relieved simultaneously.

Bile was taken on September 10, and urine was taken from September 3 to 4. TCA concentrations in bile and urine were determined by colorimetry. Bile was deproteinized with sodium tungstate and sulfuric acids and TCA in the supernatant was extracted with ethyl ether 5 times by the method described previously, ${ }^{8)}$ and the ethyl ether layer was dried under a reduced pressure. The residue was dissolved in distilled water, and TCA was determined by alkali- 
pyridine- $p$-cloroaniline colorimetry as described elsewhere. ${ }^{9)}$ Biliary trichloroethanol (TCE-OH) was determined by colorimetry after hydrolysis of TCE-OH glucronide with $\beta$ glucuronidase. ${ }^{9)}$ Urinary TCA and TCE-OH concentrations were determined by the same method used for bile analysis but with ether extraction omitted.

Concentrations of TCA in A and B biles were $2.7 \mathrm{mg} / 1$ and $3.0 \mathrm{mg} / \mathrm{l}$, respectively and those in urine were $13 \mathrm{mg} / \mathrm{g}$ creatinine and $11 \mathrm{mg} / \mathrm{g}$ creatinine for the first and second $12 \mathrm{hr}$ samples. Trace amounts of TCE-OH were found in urine, but not in bile.

The case we have presented here is considered primary PCI, not secondary PCI by adhesion of the omentum after appendicitis, because the patient's intestinal cysts disappeared and constipation was also relieved after hyperbaric oxygen inhalation therapy. Sato et al. ${ }^{7,10)}$ reported that the main locus of the cysts was the sigmoid colon in PCI patients with TRI exposure. Our patient, who also had sigmoid cysts, may be another case of primary PCI possibly related to TRI exposure.

\section{REFERENCES}

1) Jamart J. Pneumatosis cystoides intestinalis. A statistical study of 919 cases. Acta Hepato Gastroenterol 1979; 26: 419-22.

2) Koss LG. Abdominal gas cysts (Pneumatosis cystoides intestinarum hominis). Arch Pathol 1952; 53: 523-48.

3) Yamaguchi $\mathrm{K}$ et al. Twelve cases of pneumatosis cystoides intestinalis with the etiological implication of trichloroethylene. Japanese Journal of Gastroenterology 1983; 80: 1659.

4) Kurihara Y, Yokogi K, Terashima K, Suzuki S, Wada T, Igari H, Igari S, Obara K, Igarashi T, Yoshida H, Kasukawa R, Sato M, Nagai Y, Hara K. Pneumatosis cystoides intestinalis in the colon which seemed to be caused by chronic exposure to trichloroethylene. Japanese Journal of Gastorenterology. A Case Report 1985; 82: 1580-4. (In Japanese)

5) Kurihara Y, Yokogi K, Suzuki S, Wada T, Igari H, Obara K, Igarashi T, Yoshida H, Kasukawa R. A survey for the detection of trichloroacetic acid (TCA) from urine of workers exposed to perchloroethylene (PE) and clinical study of three cases with pneumatosis cytoides coli (PCC) which seemed to be caused by chronic exposure to trichloroethylene (TE). Japanese Journal of Gastroenterology 1986; 83: 1455-60. (In Japanese)

6) Arai $H$, Tanaka $M$, Horiguchi $M$, Kimura $K$, Nomiyama $H$, Nomiyama $K$, Yamaguchi $T$, Saito K. A case of pneumatosis cystoides coli with a history of occupational exposure to trichloroethylene. Japanese Journal of Gastroenterology 1985; 82: 1786-90. (In Japanese).

7) Sato A, Yamaguchi $K$, Nakajima $T$. Occupational trichloroethylene exposure causes pneumatosis cytoids intestinalis ?. Jpn J Ind Health 1986; 28: 57-62. (In Japanese)

8) Ogata M, Saeki T. Measurement of chloral hydrate, trichloroethanol, trichloroacetic acid and monochloroacetic acid in the serum and the urine by gas chromatography. Int Arch Arbeitsmed 1974; 33: 49-58.

9) Ogata M, Simada $Y$, Taguchi T. A new microdetermination method used in an analysis of the excretion of trichloro compounds in the urine of workers exposed to trichloro- 
ethylene vapour. Ind Health 1987; 25: 103-12.

10) Sato A, Yamaguchi K, Nakajima T. A new health prob'em due to trichloroethylene; Pneumatosis cystoides intestinalis. Arch of Environ Health 1987; 42: 144-7.

1) Department of Public Health, Okayama University Medical School 2-5-1 Shikata-cho, Okayama City, Okayama, 700 Japan

2) Department of Gastroenterology, Kawasaki Medical School 577 Matushima, Kurashiki City, Okayama, 701-01 Japan

3) Okayama Prefectual Research Center of Environment and Health 739-1 Uchio, Okayama City, Okayama, 700 Japan

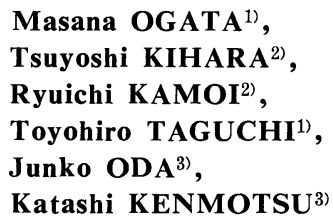

(Received November 9, 1987 and in revised from June 9, 1988) 